\title{
Combined focal myoclonus and dystonia secondary to a cerebellar hemorrhage: a case report
}

\author{
Guangxun Shen', Guangxian Nan ${ }^{1}$, Chae-Won Shin ${ }^{2}$, Hyeyoung Park ${ }^{3}$, Kwee-Yum Lee ${ }^{4}$ and Beomseok Jeon ${ }^{3 *}$
}

\begin{abstract}
Background: Myoclonus is a clinical sign characterized by sudden, brief jerky, shock-like involuntary movements of a muscle or group of muscles. Dystonia is defined as a syndrome of sustained muscle contractions, frequently causing twisting and repetitive movements or abnormal postures. Cases of myoclonus or dystonia secondary to a structural lesion in the cerebellum have been reported. However, there has never been a reported case of combined myoclonus and dystonia secondary to a cerebellar lesion.

Case presentation: Herein, we report a 22-year-old female patient with sudden-onset myoclonic jerks, dystonic posture and mild ataxia in the right upper extremity. At age 19, she experienced sudden headache with vomiting. The neurological examination showed ataxia, myoclonus and dystonia in the right upper extremity. Brain images demonstrated a hemorrhage in the right cerebellar hemisphere secondary to a cavernous malformation. After resection of the hemorrhagic mass, headache with vomiting disappeared and ataxia improved, but myoclonus and dystonia persisted.
\end{abstract}

Conclusions: It is the first report of combined focal myoclonus and dystonia secondary to a cerebellar lesion.

Keywords: Myoclonus, Dystonia, Cerebellar lesion, Magnetic resonance imaging, Electromyography

\section{Background}

Myoclonus is a clinical sign characterized by sudden, brief jerky and shock-like involuntary movements of a muscle or group of muscles. The main anatomical origins of the myoclonic jerks are known to be cortical, subcortical, spinal, and peripheral. Although rare, several cases of myoclonus secondary to a structural lesion in the cerebellum have been reported. Therefore, it has been speculated that cerebellum might be one of the possible generators of myoclonus [1]. Progressive myoclonus was reported in a young child with a ganglioglioma in the region of the deep cerebellar white matter and nuclei [2].

Dystonia is defined as a syndrome of sustained muscle contractions, frequently resulting in twisting and repetitive movements or abnormal postures. Although most

\footnotetext{
* Correspondence: brain@snu.ac.kr

${ }^{3}$ Department of Neurology, Seoul National University College of Medicine,

Seoul National University Hospital, 101 Daehak-ro, Jongno-gu, Seoul 110-774,

Republic of Korea

Full list of author information is available at the end of the article
}

common structural lesions responsible for dystonia are in basal ganglia, cerebellum is also considered to be important in dystonia [3, 4]. Dystonia secondary to a cerebellar lesion is rare, but several cases have been reported in the literature. For example, focal limb dystonia was demonstrated in a patient with a cerebellar mass [5]. Herein, we describe an unusual case of sudden-onset combined focal myoclonus and dystonia secondary to a cerebellar lesion.

\section{Case presentation}

A 22-year-old right-handed woman was referred for management of jerky movements and abnormal posturing of the right hand that had developed since acute hemorrhage in the right cerebellar hemisphere secondary to a cavernous malformation. At age 19, she developed an episode of severe headache with vomiting, and poor coordination. Examination revealed ataxia in her right upper extremity along with jerks and abnormal posturing of her right hand and fingers. Brain computed tomography (CT) and magnetic resonance imaging (MRI) showed a hemorrhage in 
the right cerebellar hemisphere compressing the fourth ventricle (Fig. 1a and b). No structure lesions were found in the supratentorial region. Resection of the mass and pathological examination confirmed the diagnosis of cavernous malformation. Her headache improved after surgery. However, mild ataxia in the right arm, abnormal posturing of the hand and spontaneous and sudden jerks of the right fingers persisted. Furthermore, the jerks were unable to be suppressed voluntarily but disappeared during sleep. Alcohol intake did not seem to affect those abnormal movements, but anxiety and stress worsened the symptoms.

Neurological examination at our clinic showed mild dysmetria in the right upper extremity on the finger-tonose test. The right hand showed occasional brisk jerks at rest which became more evident on maintaining posture, but were not provoked by touch. Abnormal posturing of the fingers was noted, which was accentuated while outstretching. An abnormally tight grip was observed while writing with a pencil. (See Additional file 1, a video footage of the patient showing these features in more detail). The patient presented symmetrical deep tendon reflex and intact proprioceptive sensation. No long-tract signs were found. There were no changes in the severity of these involuntary movements during a 6-month trial of piracetam, biperiden, baclofen and clonazepam.

A 15-month follow-up MRI demonstrated a postoperative malacic lesion in the right cerebellar hemisphere (Fig. 1c). Surface electromyography (EMG) recordings of the right forearm while keeping outstretched illustrated the typical features of myoclonus with synchronous bursts (93-97 ms) of EMG activities in agonist and antagonist muscles (Fig. 2).

\section{Conclusion}

Our patient presented with sudden-onset shock-like jerks in her right fingers, superimposed on more sustained abnormality of posture in the right hand with mild ataxia in the right arm. Surface EMG recordings confirmed the jerks as myoclonus, which were synchronous and short-lasting activities in agonist and antagonist muscles. Dystonic posturing of the right hand was evident in clinical examination (Additional file 1). It is most likely that the cerebellar lesion is responsible for both myoclonus and dystonia in our case based on the following reasons: 1) The onset of the myoclonus and dystonia coincided with onset of ataxia; 2) The lesion
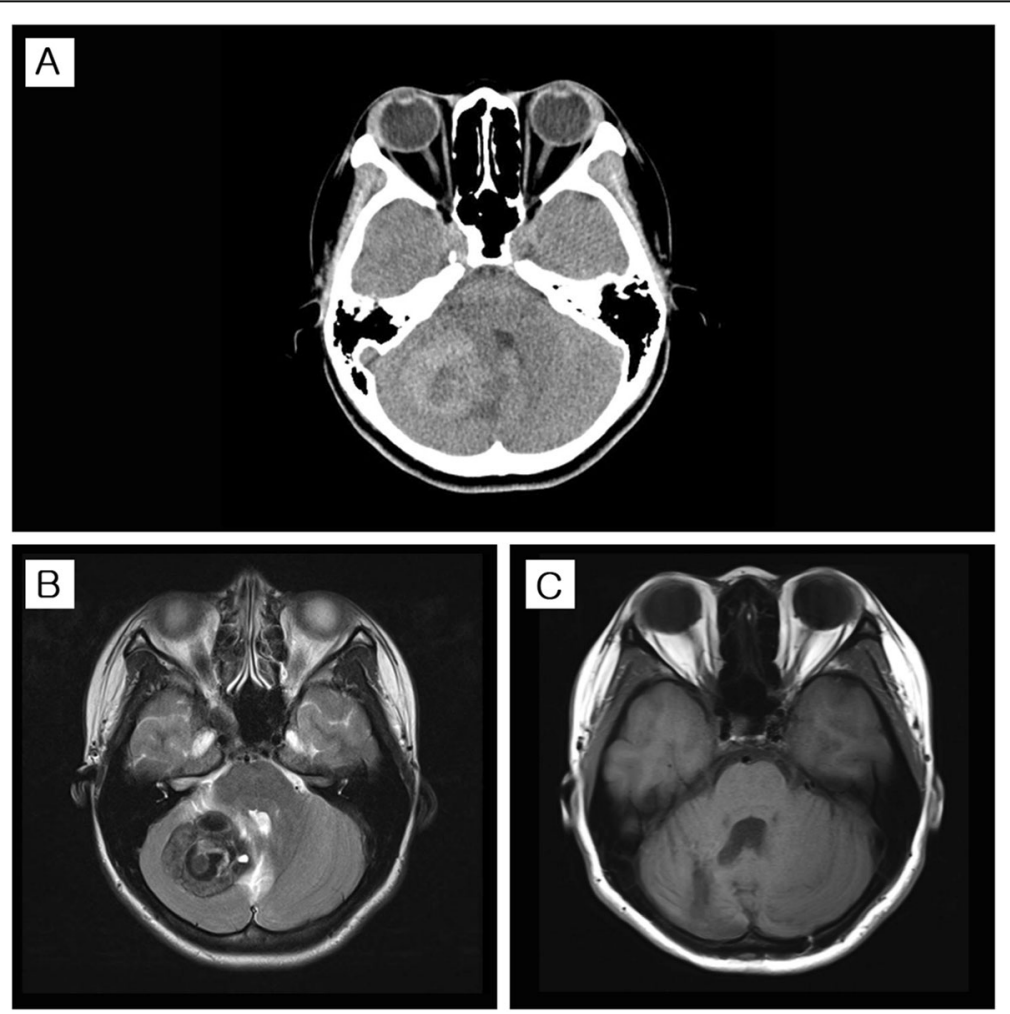

Fig. 1 Brain images. Axial CT scan at the onset of the symptoms demonstrated a hemorrhagic mass in the right cerebellar hemisphere and the fourth ventricle compressed by the mass (a). Axial T2-weighted MRI demonstrated heterogeneous low signal intensity of the mass ( $44 \times 37 \mathrm{~mm}$ ) with high signal intensity in the periphery (b). Axial T1-weighted MRI at 15-month follow-up after resection demonstrated a post-operative malacic lesion in the right cerebellar hemisphere (c) 


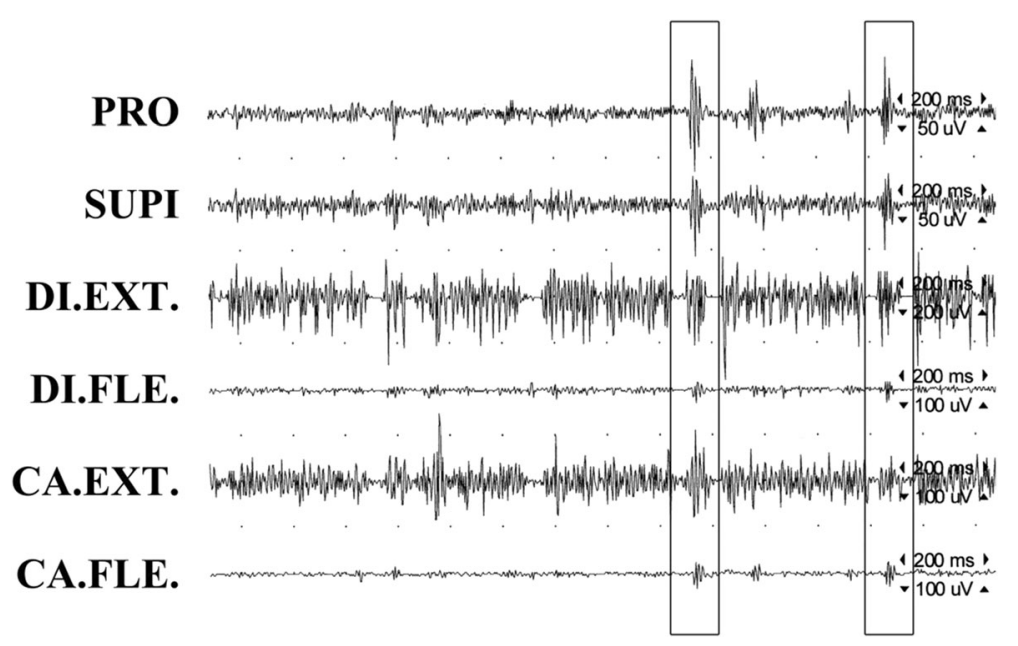

Fig. 2 Surface EMG. EMG recordings of the right upper extremity while maintaining a posture demonstrate two consecutive jerks (93-97 ms), showing synchronous bursts in agonist and antagonist muscles. PRO: pronator teres muscle; SUPI: supinator muscle; DI. EXT: extensor digitorum communis muscle; DI. FLE: flexor digitorum profundus muscle; CA. EXT: extensor carpi ulnaris muscle; CA. FLE: flexor carpi radialis muscle

of the right cerebellar hemisphere was demonstrated on the brain CT and MRI; and 3) Involuntary movements were ipsilateral to the cerebellar lesion.

Cerebellum as a structure in cerebro-cerebellar loops, participates in motor control by means of sensorimotor integration - the process whereby sensory input is integrated by the central nervous system and used for assisting motor program execution $[6,7]$.

Even though there have been reports of dystonia in cerebellar lesion, the pathophysiology is not entirely clear. However, there are suggestions that abnormal cerebellar learning and functional interactions between cerebellar and basal ganglia circuits may play a role $[8,9]$.

Role of cerebellum in myoclonus is also not clear. However, there are several reported cases of cortical myoclonus in patients with cerebellar pathology, which are documented by neurophysiology and pathology [10]. This paradoxical absence of cortical pathology and cortical myoclonus in patients with cerebellar pathology has promoted the authors to suggest that enhanced excitability of the sensorimotor cortex may arise as a distant effect of cerebellar pathology [10]. This has further been explored in the review by Ganos et al. [11].

Our case is unique in that the hemorrhage in the right cerebellar hemisphere and subsequent surgical operation result in myoclonus and dystonia at the same time, and it further supports the role of cerebellum in the generation of myoclonus and dystonia.

In conclusion, the findings in our patient support that movement disorders resulting from lesions of the cerebellum may include myoclonus and dystonia.

\section{Additional file}

Additional file 1: A 22-year-old woman with focal myoclonus and dystonia in the right upper extremity. No myoclonus is noted at rest or triggered by external stimuli. Postural myoclonus of the right hand is prominent and her right fingers show dystonic posturing when she outstretches the arms and rotates the wrist. The abnormal posturing of hand also interferes with a writing task. The patient shows normal gait. (MP4 $32.1 \mathrm{mb}$ )

\section{Abbreviations}

CT: Computed tomography; EMG: Electromyography; MRI: Magnetic resonance imaging

\section{Acknowledgments}

We thank the patient and her family for their consents to publish the case report and the accompanying video segment.

Funding

We receive no funding support.

Availability of data and materials

All data and material (Additional file 1: video) supporting our findings are contained within the manuscript.

\section{Authors' contributions}

GS examined the patient and drafted the manuscript. GN evaluated the neuroimaging findings and provided critical clinical opinions. CWS and HP helped with collection and analysis of EMG and clinical data. KYL helped with English-editing of the manuscript. BJ conceived the case and is accountable for the integrity of the entire work. All authors read and approved the final manuscript.

\section{Competing interests}

The author (s) declared no conflicts of interest with respect to the research, authorship, funding, and/or publication of this article.

\section{Consent for publication}

Written informed consent was obtained from the patient and patient's mother for publication of this case report and accompanying images. A copy of the written consent is available for review by the Editor-in-Chief of this journal. 


\section{Ethics approval and consent to participate}

The patient has given her consent for this case report.

\section{Author details}

'Department of Neurology, China-Japan Union Hospital of Jilin University, Changchun, China. ${ }^{2}$ Department of Neurology, SNU-SMG Boramae Medical Center, Seoul, Republic of Korea. ${ }^{3}$ Department of Neurology, Seoul National University College of Medicine, Seoul National University Hospital, 101 Daehak-ro, Jongno-gu, Seoul 110-774, Republic of Korea. ${ }^{4}$ School of Medicine, University of Queensland, Brisbane, QLD 4072, Australia.

\section{Received: 12 July 2016 Accepted: 8 November 2016}

\section{Published online: 17 November 2016}

\section{References}

1. Koh KN, Lim BC, Hwang H, Park JD, Chae JH, Kim KJ, Hwang YS, Kim SK, Wang KC, Moon HK. Cerebellum can be a possible generator of progressive myoclonus. J Child Neurol. 2010;25:728-31.

2. Mink JW, Caruso PA, Pomeroy SL. Progressive myoclonus in a child with a deep cerebellar mass. Neurology. 2003;61:829-31.

3. Prudente CN, Hess EJ, Jinnah HA. Dystonia as a network disorder: what is the role of the cerebellum? Neuroscience. 2014;260:23-35.

4. Quartarone A, Hallett M. Emerging concepts in the physiological basis of dystonia. Mov Disord. 2013;28:958-67.

5. Alarcón F, Tolosa E, Muñoz E. Focal limb dystonia in a patient with cerebellar mass. Arch Neurol. 2001:58:1125-7.

6. Proville RD, Spolidoro M, Guyon N, Dugué GP, Selimi F, Isope P, Popa D, Léna C. Cerebellum involvement in cortical sensorimotor circuits for the control of voluntary movements. Nat Neurosci. 2014;17(9):1233-9.

7. Popa D, Spolidoro M, Proville RD, Guyon N, Belliveau L, Léna C. Functional role of the cerebellum in gamma-band synchronization of the sensory and motorcortices. J Neurosci. 2013;33(15):6552-6.

8. Hoffland BS, Veugen LC, Janssen MM, Pasman JW, Weerdesteyn V, van de Warrenburg BP. A gait paradigm reveals different patterns of abnormal cerebellar motor learning in primary focal dystonias. Cerebellum. 2014;13(6):760-6.

9. Neychev VK, Fan X, Mitev VI, Hess EJ, Jinnah HA. The basal ganglia and cerebellum interact in the expression of dystonic movement. Brain. 2008:131(Pt 9):2499-509.

10. Tijssen MA, Thom M, Ellison DW, Wilkins P, Barnes D, Thompson PD, Brown P. Cortical myoclonus and cerebellar pathology. Neurology. 2000;54(6):1350-6.

11. Ganos C, Kassavetis P, Erro R, Edwards MJ, Rothwell J, Bhatia KP. The role of the cerebellum in the pathogenesis of cortical myoclonus. Mov Disord. 2014:29(4):437-43.

\section{Submit your next manuscript to BioMed Central and we will help you at every step:}

- We accept pre-submission inquiries

- Our selector tool helps you to find the most relevant journal

- We provide round the clock customer support

- Convenient online submission

- Thorough peer review

- Inclusion in PubMed and all major indexing services

- Maximum visibility for your research

Submit your manuscript at www.biomedcentral.com/submit
Biomed Central 